\title{
One-loop flavor changing electromagnetic transitions
}

\author{
Q. Ho-Kim ${ }^{1}$ and X. Y. Pham ${ }^{2}$ \\ ${ }^{1}$ Department of Physics, Université Laval \\ Ste-Foy (QC) Canada G1K $7 P_{4}$ \\ e-mail: qhokim@phy.ulaval.ca \\ ${ }^{2}$ Laboratoire de Physique Théorique et Hautes Energies \\ Universités Pierre et Marie Curie $\&$ Denis Diderot \\ F-75252 Paris Cedex 05 France \\ e-mail: pham@lpthe.jussieu.fr
}

\begin{abstract}
We discuss the effect of the external fermion masses in the flavorchanging radiative transitions of a heavy fermion (quark or lepton) to a lighter fermion at the one-loop level, and point out an often overlooked crucial difference in the sign of a charge factor between transitions of the down type $s \rightarrow d \gamma$ and the up type $c \rightarrow u \gamma$. We give formulas for the $F \rightarrow f \gamma$ effective vertex in various approximations and the exact formula for $t \rightarrow c \gamma$ and $\tau \rightarrow \mu \gamma$.

PACS numbers: 12.15.Lk, 13.40.Ks, 13.90.+i
\end{abstract}




\section{INTRODUCTION}

Flavor-changing radiative transitions $F \rightarrow f \gamma$ are processes in which a fermion (quark or lepton) undergoes a flavor change accompanied by the emission of a real or virtual photon. They are the results of an interplay of the weak and electromagnetic interactions at the loop level, often enhanced by QCD effects. As such, they have considerable theoretical and experimental interest, not only because they provide excellent tests of the standard model but also because they hold the promise of being sensitive to new physics. They are operating in many different situations, for example in radiative weak decays of hyperons, such as $\Sigma^{+} \rightarrow p \gamma$ and $\Xi^{0} \rightarrow \Lambda \gamma$, in rare $B$ meson decays $\left(B \rightarrow X_{s} \gamma\right)$, and in rare $D$ meson decays $(D \rightarrow V \gamma)$. They may also occur in rare processes involving leptons, such as $K \rightarrow \pi e^{+} e^{-}, \tau \rightarrow \mu \gamma$, and $\nu_{1} \rightarrow \nu_{2} e^{+} e^{-}$.

At the quark or lepton level, all flavor-changing electromagnetic transitions may be divided into two categories: one involving the upper components of weak isospin doublets and the other their lower components, as shown in Table

1. In one loop processes, the initial fermion $F$ creates an intermediate state of a boson and a fermion $f_{\ell}$ which, after emitting a photon, returns to a fermionic state $f$ as depicted in Figs. 1 and 2 for an example of quark transition.

\begin{tabular}{lll}
\hline \hline$F$ & $f$ & $f_{\ell}$ \\
\hline$\nu_{1}$ & $\nu_{2}$ & $e^{-}, \mu^{-}, \tau^{-}$ \\
$c$ & $u$ & $d, s, b$ \\
$t$ & $c(u)$ & $d, s, b$ \\
\hline$\tau^{-}$ & $\mu^{-}\left(e^{-}\right)$ & $\nu_{j}, j=1,2,3$ \\
$s$ & $d$ & $u, c, t$ \\
$b$ & $s(d)$ & $u, c, t$ \\
\hline \hline
\end{tabular}

Table 1: Elementary flavor-changing radiative processes.

The amplitudes of these penguin processes have been evaluated many years ago [1, 2]. In particular, a simple analytic formula obtained by Inami and Lim [1] has often served as the starting point for several more advanced calculations of QCD corrections [3]- [5] or properties of detailed particle models [5]- 6]. The interested reader is referred to a recent review [7] for further discussion.

Unfortunately several authors have overlooked the fact that this formula applies only to amplitudes of the down-type $s d \gamma$, which were the primary object of interest to Inami and Lim [1], and have used it unchanged to study processes of the up-type $c u \gamma$. As we shall see, this oversight will cause an error in sign and magnitude (by a factor of five) in the amplitudes of the up-type transitions. Moreover, in the down-type $b \rightarrow s \gamma$ transition, the intermediate state is dominated by the contribution of the top quark and therefore it is well justified to neglect the effects of the external fermion masses as it was done before [1], but it is doubtful that such an approximation will hold in other cases, especially in $c \rightarrow u \gamma$ and $\tau \rightarrow \mu \gamma$, not to mention $t \rightarrow c \gamma$, in which the mass of the initial 
particle is comparable or larger than any internal (boson or quark) mass.

In this paper, we will correct the above-mentioned shortcomings and proceed on to examine the effect of the external fermionic masses in flavor-changing radiative transitions. After writing down the rules and conventions of calculations in Sec. II, we will evaluate in Sec. III the effective vertex for $F \rightarrow f \gamma$ assuming first small but non-vanishing external fermionic masses. Although the methods we follow are applicable to both quarks and leptons, we refer specifically to quarks for convenience. We calculate the inclusive rates for $q_{i} \rightarrow q_{j} \gamma$ and examine the effects of the external masses on the decay rates in some detail; we also give an estimate of the QCD-corrected branching ratio of $c \rightarrow u \gamma$. In Sec. IV, we derive an exact formula, with $m_{c}=0$ but non-vanishing $m_{t}$, for the effective $t \rightarrow c \gamma$ vertex and demonstrate the importance of the initial quark mass. We conclude with Sec. V.

\section{FEYNMAN RULES IN THE't HOOFT-FEYNMAN GAUGE}

The standard electroweak model formulated in any renormalizable gauge $R_{\xi}$ contains, besides the usual physical particles (the photon $A_{\mu}$, bosons $\mathrm{W}_{\mu}^{ \pm}$, and quarks $\mathrm{q}_{i}$ ), the unphysical would-be Goldstone scalar bosons $\varphi^{ \pm}, \varphi^{0}$ coming from the spontaneous symmetry breaking (as well as the Faddeev-Popov ghosts $\chi^{ \pm}, \chi^{0}$ which, however, do not contribute to processes considered here because the external particles are fermions). In writing down the following Feynman rules we use the convention that defines ingoing momenta for particles entering the vertex and outgoing momenta for particles leaving the vertex. Quark flavors will be denoted by $i$ or $j$, and the left and right projection operators by $L=$ $\left(1-\gamma_{5}\right) / 2$ and $R=\left(1+\gamma_{5}\right) / 2$.

The rules for the elementary vertices are

$$
\begin{aligned}
& q_{i} \rightarrow q_{i} A_{\mu}:-i e Q_{i} \gamma_{\mu} \quad \text { with } Q_{i}=\frac{2}{3}(u),-\frac{1}{3}(d), \\
& W_{\lambda}^{ \pm}(p) \rightarrow W_{\nu}^{ \pm}\left(p^{\prime}\right) A_{\mu}:+i e Q_{W}\left[\left(p+p^{\prime}\right)_{\mu} g_{\lambda \nu}+\left(p^{\prime}-2 p\right)_{\nu} g_{\lambda \mu}\right. \\
&\left.\quad \quad+\left(p-2 p^{\prime}\right)_{\lambda} g_{\mu \nu}\right] \quad \text { with } Q_{W}= \pm 1\left(W^{ \pm}\right), \\
& \varphi^{ \pm}(p) \rightarrow \varphi^{ \pm}\left(p^{\prime}\right) A_{\mu}:-i e Q_{S}\left(p+p^{\prime}\right)_{\mu} \quad \text { with } Q_{S}= \pm 1\left(\varphi^{ \pm}\right), \\
& W_{\nu}^{ \pm} \rightarrow \varphi^{ \pm} A_{\mu}:+i e M_{W} g_{\mu \nu}, \\
& q_{i} \rightarrow q_{j} W_{\mu}^{ \pm}: \frac{-i g}{\sqrt{2}} \gamma_{\mu} L U_{j i}^{\left(I_{i}\right)}, \\
& q_{i} \rightarrow q_{j} \varphi^{ \pm}: \frac{-i g}{\sqrt{2} M_{W}} I_{i}\left(m_{j} L-m_{i} R\right) U_{j i}^{\left(I_{i}\right)} .
\end{aligned}
$$

The symbol $I_{i}$ which appears in the last two rules is related to the weak isospin of the initial quark. For $I_{i}=+1$ ( $u$-type quark) the CKM matrix

element is $U_{j i}^{(+)}=V_{i j}^{*}$, and for $I_{i}=-1$ ( $d$-type quark) $U_{j i}^{(-)}=V_{j i}$. Note that $I_{i}=Q_{W}\left(\right.$ or $\left.Q_{S}\right)$ and thus, with the exception of the $\varphi A W$ and $q q W$ vertices, all 
other vertices depend on the sign of the charge of the quark or boson involved. Finally, $e$ is the positive unit of the electric charge, $e>0$.

The relevant boson propagators in the 't Hooft-Feynman $(\xi=1)$ gauge are

$$
\begin{array}{ll}
\frac{i}{p^{2}-M_{W}^{2}} & \text { (scalar), } \\
\frac{-i g_{\mu \nu}}{p^{2}-M_{W}^{2}} & \text { (W boson). }
\end{array}
$$

\section{CONTRIBUTIONS TO THE EFFECTIVE VERTEX}

In the lowest order, i.e. at the one-loop level, the processes in which a quark undergoes a flavor change accompanied by the emission of a real or virtual photon are represented by the diagrams in Figs. 1(a)-(d) and Figs. 2(a)-(b). In the first group, the photon is emitted by a scalar or a vector boson in the intermediate state, whereas in the second it arises from an internal fermion. As we are eventually interested in real, transversally polarized photon emission, the transition should take place in a magnetic mode characterized by the operator $i \sigma_{\mu \nu} q^{\nu}$, and we need not concern ourselves with diagrams in Fig. 3 since they are all proportional to $\gamma_{\mu}$.

We perform our calculations in $n$ dimensions and let $n=4-2 \omega$. For convenience we also introduce an arbitrary scale parameter with the dimension of mass $\mu$ so that the coupling constant $g$ remains dimensionless even in arbitrary $n$ dimensions. The kinematic variables to be used are defined in Fig. 4 . In particular, the initial quark has momentum $P$ and mass $M$; the final quark has momentum $p$ and mass $m$. The internal quark, with mass $m_{\ell}$, may have flavors $u, c$, or $t$ for an initial quark of the $d$-type, and $s, d$, or $b$ for an initial quark of the $u$-type. It is understood that the various partial or total vertex operators obtained in the following for $q_{i} \rightarrow q_{f} \gamma$ are to be inserted between the initial state $u_{i}(P)$ and the final state $\bar{u}_{f}(p) \varepsilon^{\mu *}(q)$, where $u_{i}$ and $u_{f}$ are the fermion spinors, and $\varepsilon^{\mu}(q)$ is the photon polarization vector with momentum $q=P-p$. A summation over all allowed quark flavors $\ell$ and an integration over the loop $n$-dimensional $k$ momentum are both implicit.

From the rules given above, the expression corresponding to Fig. 1(a) is

$$
i T_{1 \mathrm{a}}^{\mu}=\mu^{2 \omega}\left(\frac{-i g}{\sqrt{2}}\right)^{2} \lambda_{\ell} \gamma^{\rho} L \frac{i}{k-m_{\ell}} \gamma^{\sigma} L \frac{(-i)^{2}\left(i e Q_{W} X_{\rho \sigma}^{\mu}\right)}{\left[(p-k)^{2}-M_{W}^{2}\right]\left[(P-k)^{2}-M_{W}^{2}\right]},
$$

where the following shorthand notations have been used:

$$
X_{\mu \rho \sigma}=(P+p-2 k)_{\mu} g_{\rho \sigma}+(P+k-2 p)_{\sigma} g_{\rho \mu}+(p+k-2 P)_{\rho} g_{\mu \sigma}
$$

and

$$
\begin{array}{rll}
i=u \text { type }: & Q_{W}=+1, \lambda_{\ell}=V_{f \ell} V_{i \ell}^{*} \\
i=d \text { type }: & Q_{W}=-1, \lambda_{\ell}=V_{\ell f}^{*} V_{\ell i} .
\end{array}
$$


It is convenient to put (11) in the form

$$
i T_{1 \mathrm{a}}^{\mu}=\frac{-g^{2} e}{2} \mu^{2 \omega} Q_{W} \lambda_{\ell} \frac{N_{1 \mathrm{a}}^{\mu}}{D_{1}}
$$

with the following expressions for the numerator and denominator:

$$
\begin{aligned}
N_{1 \mathrm{a}}^{\mu} & =\gamma^{\rho} L\left(k+m_{\ell}\right) \gamma^{\sigma} L X_{\rho \sigma}^{\mu}, \\
D_{1} & =\left(k^{2}-m_{\ell}^{2}\right)\left[(p-k)^{2}-M_{W}^{2}\right]\left[(P-k)^{2}-M_{W}^{2}\right] .
\end{aligned}
$$

Corresponding to Fig. 1(b) is the transition operator

$$
\begin{aligned}
i T_{1 \mathrm{~b}}^{\mu}= & \mu^{2 \omega} \lambda_{\ell}\left[\frac{i g Q_{S}}{\sqrt{2} M_{W}}\left(m L-m_{\ell} R\right)\right] \frac{i}{\not k-m_{\ell}}\left[\frac{-i g Q_{S}}{\sqrt{2} M_{W}}\left(m_{\ell} L-M R\right)\right] \\
& \times \frac{i^{2}\left(-i e Q_{S}\right)(P+p-2 k)^{\mu}}{\left[(p-k)^{2}-M_{W}^{2}\right]\left[(P-k)^{2}-M_{W}^{2}\right]} \\
= & \frac{-g^{2} e}{2} \mu^{2 \omega} Q_{S} \lambda_{\ell} \frac{N_{1 \mathrm{~b}}^{\mu}}{D_{1}} .
\end{aligned}
$$

From now on, if the initial quark flavor $q_{i}$ is of the $u$-type, $Q_{S}=+1$; otherwise $Q_{S}=-1$. The $\lambda_{\ell}$ always follows the rules of Eq. (3). The expression for the denominator $D_{1}$ remains the same as defined above, whereas in the numerator one has

$$
N_{1 \mathrm{~b}}^{\mu}=\frac{1}{M_{W}^{2}}\left(m L-m_{\ell} R\right)\left(\not k+m_{\ell}\right)\left(m_{\ell} L-M R\right)(P+p-2 k)^{\mu} .
$$

For Fig. 1(c), the Feynman rules yield

$$
\begin{aligned}
i T_{1 \mathrm{c}}^{\mu}= & \mu^{2 \omega} \lambda_{\ell}\left(\frac{-i g}{\sqrt{2}} \gamma_{\mu} L\right) \frac{i}{k-m_{\ell}}\left[\frac{-i g Q_{S}}{\sqrt{2} M_{W}}\left(m_{\ell} L-M R\right)\right] \\
& \times \frac{\left(i e M_{W}\right)(-i)(i)}{\left[(p-k)^{2}-M_{W}^{2}\right]\left[(P-k)^{2}-M_{W}^{2}\right]} \\
= & \frac{-g^{2} e}{2} \mu^{2 \omega} Q_{S} \lambda_{\ell} \frac{N_{1 \mathrm{c}}^{\mu}}{D_{1}}
\end{aligned}
$$

with the numerator given by

$$
N_{1 \mathrm{c}}^{\mu}=\gamma^{\mu} L\left(k+m_{\ell}\right)\left(M R-m_{\ell} L\right) .
$$

The diagram in Fig. 1(d) is similar to that in Fig. 1(c), with the scalar and vector bosons exchanging roles in the intermediate state. The corresponding vertex operator is

$$
\begin{aligned}
i T_{1 \mathrm{~d}}^{\mu}= & \mu^{2 \omega} \lambda_{\ell}\left[\frac{+i g Q_{S}}{\sqrt{2} M_{W}}\left(m L-m_{\ell} R\right)\right] \frac{i}{\not k-m_{\ell}}\left(\frac{-i g}{\sqrt{2}} \gamma_{\mu} L\right) \\
& \times \frac{\left(i e M_{W}\right)(i)(-i)}{\left[(p-k)^{2}-M_{W}^{2}\right]\left[(P-k)^{2}-M_{W}^{2}\right]} \\
= & \frac{-g^{2} e}{2} \mu^{2 \omega} Q_{S} \lambda_{\ell} \frac{N_{1 \mathrm{~d}}^{\mu}}{D_{1}}
\end{aligned}
$$


with the following expression in the numerator

$$
N_{1 \mathrm{~d}}^{\mu}=\left(m L-m_{\ell} R\right)\left(\not k+m_{\ell}\right) \gamma^{\mu} L .
$$

The photon may be emitted also from the intermediate quark as shown in Figs. 2(a)-(b). The diagram in Fig. 2(a) represents the transition operator

$$
\begin{aligned}
i T_{2 \mathrm{a}}^{\mu}= & \mu^{2 \omega} \lambda_{\ell}\left(\frac{-i g}{\sqrt{2}} \gamma^{\rho} L\right) \frac{i}{\not p-k-m_{\ell}}\left(-i e Q_{\ell} \gamma^{\mu}\right) \\
& \times \frac{i}{P-\not k-m_{\ell}}\left(\frac{-i g}{\sqrt{2}} \gamma_{\rho} L\right) \frac{-i}{k^{2}-M_{W}^{2}} \\
= & \frac{-g^{2} e}{2} \mu^{2 \omega} Q_{\ell} \lambda_{\ell} \frac{N_{2 \mathrm{a}}^{\mu}}{D_{2}},
\end{aligned}
$$

where $N_{2 \mathrm{a}}^{\mu}$ and $D_{2}$ stand for

$$
\begin{aligned}
N_{2 \mathrm{a}}^{\mu} & =\gamma^{\rho}\left(\not p-\not k+m_{\ell}\right) \gamma^{\mu}\left(\not p-\not k+m_{\ell}\right) \gamma_{\rho} L, \\
D_{2} & =\left(k^{2}-M_{W}^{2}\right)\left[(p-k)^{2}-m_{\ell}^{2}\right]\left[(P-k)^{2}-m_{\ell}^{2}\right] .
\end{aligned}
$$

Similarly for Fig. 2(b), we have

$$
\begin{aligned}
i T_{2 \mathrm{~b}}^{\mu}= & \mu^{2 \omega} \lambda_{\ell}\left[\frac{+i g Q_{S}}{\sqrt{2} M_{W}}\left(m L-m_{\ell} R\right)\right] \frac{i}{\not p-\not k-m_{\ell}}\left(-i e Q_{\ell} \gamma^{\mu}\right) \\
& \times \frac{i}{p-\not k-m_{\ell}}\left[\frac{-i g Q_{S}}{\sqrt{2} M_{W}}\left(m_{\ell} L-M R\right)\right] \frac{i}{k^{2}-M_{W}^{2}} \\
= & \frac{-g^{2} e}{2} \mu^{2 \omega} Q_{\ell} \lambda_{\ell} \frac{N_{2 \mathrm{~b}}^{\mu}}{D_{2}}
\end{aligned}
$$

together with the expression for the numerator

$$
N_{2 \mathrm{~b}}^{\mu}=\frac{1}{M_{W}^{2}}\left(m L-m_{\ell} R\right)\left(\not p-\not k+m_{\ell}\right) \gamma^{\mu}\left(\not P-\not k+m_{\ell}\right)\left(m_{\ell} L-M R\right) .
$$

Thus, all partial contributions to the effective radiative vertex may be written in the form

$$
i T_{p \alpha}^{\mu}=\frac{-g^{2} e}{2} \mu^{2 \omega} Q_{p} \lambda_{\ell} \frac{N_{p \alpha}^{\mu}}{D_{p}} \quad(p=1,2 ; \alpha=a, b, \ldots)
$$

with the charge factor

$$
Q_{1}=Q_{B}\left(Q_{W} \text { or } Q_{S}\right), \quad Q_{2}=Q_{\ell}
$$

After Feynman parameterization of the denominators, the integration over $n$-dimensional momentum space is performed in the standard way. Consider for example $T_{1 \mathrm{a}}^{\mu}$. The terms independent of the integrated momentum $k$ are evaluated as follows:

$$
\begin{aligned}
\mu^{2 \omega} \int \frac{\mathrm{d}^{n} k}{(2 \pi)^{n}} \frac{1}{D_{1}} & =\mu^{2 \omega} \int \frac{\mathrm{d}^{n} k}{(2 \pi)^{n}} \int_{0}^{1} \mathrm{~d} x \int_{0}^{1-x} \mathrm{~d} y \frac{2}{[a(1-x-y)+b x+c y]^{3}} \\
& =\frac{-i}{(4 \pi)^{2}} \int_{0}^{1} \mathrm{~d} x \int_{0}^{1-x} \mathrm{~d} y \frac{1}{M_{W}^{2} \Lambda_{1}}\left(\frac{4 \pi \mu^{2}}{-M_{W}^{2} \Lambda_{1}}\right)^{\omega} \Gamma(1+\omega)
\end{aligned}
$$


for $a=(p-k)^{2}-M_{W}^{2}, b=k^{2}-m_{\ell}^{2}, c=(P-k)^{2}-M_{W}^{2}$, and

$$
\begin{aligned}
M_{W}^{2} \Lambda_{1} & =M_{W}^{2}(1-x)+m_{\ell}^{2} x-P^{2} x y-p^{2} x z-q^{2} y z \\
z & =1-x-y .
\end{aligned}
$$

Terms with explicit $k$-dependent integrands are handled in a similar way. With the summation over the internal quarks now explicit, the $k$ integration therefore carries Eq. (11) into

$$
\begin{aligned}
i T_{1 \mathrm{a}}^{\mu}= & \frac{-g^{2} e}{2} Q_{W} \mu^{2 \omega} \sum_{\ell} \lambda_{\ell} \int \frac{\mathrm{d}^{n} k}{(2 \pi)^{n}} \frac{N_{1 \mathrm{a}}^{\mu}}{D_{1}} \\
= & \frac{i g^{2} e}{32 \pi^{2} M_{W}^{2}} Q_{W} \sum_{\ell} \lambda_{\ell} \int_{0}^{1} \mathrm{~d} x \int_{0}^{1-x} \mathrm{~d} y \frac{1}{\Lambda_{1}} \\
& \times R\left\{\left[-4 x P^{\mu}+2(1-2 z) q^{\mu}\right][(1-x) \not P-z \not q]\right. \\
& +(1-x)\left(\not q \gamma^{\mu} \not P-\not P \gamma^{\mu} \not q\right)+(-x \not P-z \not q)[(1-x) \not P-z \not q] \gamma^{\mu} \\
& \left.+\gamma^{\mu}[(1-x) \not P-z \not q][-x \not P+(1-z) \not q]+4(1-n) \gamma^{\mu} \mathcal{F}_{1}\right\}
\end{aligned}
$$

Here $\mathcal{F}_{1}$ comes from the singular part of the quadratic $k$-dependent integrand. This result will considerably simplify when the external particles go on shell, which is all we need. When the initial and final quarks are on the mass shell, we may use the Dirac equations $P u_{i}(P)=M u_{i}(P)$ and $\bar{u}_{f}(p) \not p=m \bar{u}_{f}(p)$ to express the operator $T_{1 \mathrm{a} \mu}$ in terms of the four-vectors $P_{\mu}, q_{\mu}$, and $\gamma_{\mu}$ or, alternatively, $i \sigma_{\mu \nu} q^{\nu}, q_{\mu}$, and $\gamma_{\mu}$, the two bases being related by

$$
\begin{aligned}
& 2 P_{\mu} R=R\left(i \sigma_{\mu \nu} q^{\nu}+q_{\mu}+M \gamma_{\mu}\right)+L m \gamma_{\mu}, \\
& 2 P_{\mu} L=L\left(i \sigma_{\mu \nu} q^{\nu}+q_{\mu}+M \gamma_{\mu}\right)+R m \gamma_{\mu} .
\end{aligned}
$$

These relations are understood as being sandwiched between $\bar{u}(p)$ and $u(P)$. The induced complete $q_{i} q_{f} \gamma$ vertex will then assume the general form

$$
T_{\mu} \sim R\left(q^{2} \gamma_{\mu}-q_{\mu} \not q\right) F+i \sigma_{\mu \nu} q^{\nu}(R M G+L m H),
$$

where $F, G$, and $H$ are appropriate form factors. For an on-shell photon, $q^{2}=0$, we may set $q_{\mu} \varepsilon^{\mu}(q)=0$, and the coefficient of $F$ should vanish. The singular terms, such as $\mathcal{F}_{1}$, from all diagrams likewise completely cancel out [8]. Hence, with real photon radiative processes in mind, we may drop all such terms in the individual contributions. When the above simplifications are applied, $T_{1 \mathrm{a}}^{\mu}$ reduces on the mass shell to

$$
\begin{aligned}
T_{1 \mathrm{a}}^{\mu}= & \frac{e G_{\mathrm{F}}}{4 \sqrt{2} \pi^{2}} Q_{\mathrm{W}} \sum_{\ell} \lambda_{\ell} \int_{0}^{1} \mathrm{~d} x \int_{0}^{1-x} \mathrm{~d} y \frac{1}{\Lambda_{1}} \\
& \times\{R M[1-x+y(1-2 x)]+\operatorname{Lm}[1-x+z(1-2 x)]\} i \sigma^{\mu \nu} q_{\nu},
\end{aligned}
$$

where $G_{\mathrm{F}} / \sqrt{2}=g^{2} /\left(8 M_{W}^{2}\right)$ and

$$
\Lambda_{1}=1-x+r_{\ell} x-r_{i} x y-r_{f} x z
$$


with $r_{\ell}=m_{\ell}^{2} / M_{W}^{2}, r_{i}=M^{2} / M_{W}^{2}$, and $r_{f}=m^{2} / M_{W}^{2}$. The contributions from all the other diagrams may be reduced to this standard form.

Let us sum up the partial contributions in each group $(p=1,2)$ of diagrams to get the final transition operators

$$
T_{p}^{\mu}=\frac{e G_{\mathrm{F}}}{4 \sqrt{2} \pi^{2}} Q_{p} \sum_{\ell} \lambda_{\ell}\left[R M G_{p}\left(r_{\ell}\right)+L m H_{p}\left(r_{\ell}\right)\right] i \sigma^{\mu \nu} q_{\nu},
$$

where we have used $Q_{p}$ defined in (17), and introduced

$$
\begin{aligned}
G_{p}\left(r_{\ell}\right) & =\int_{0}^{1} \mathrm{~d} x \int_{0}^{1-x} \mathrm{~d} y \frac{1}{\Lambda_{p}} g_{p}\left(r_{\ell}, x, y\right), \\
H_{p}\left(r_{\ell}\right) & =\int_{0}^{1} \mathrm{~d} x \int_{0}^{1-x} \mathrm{~d} y \frac{1}{\Lambda_{p}} h_{p}\left(r_{\ell}, x, y\right) \quad(p=1,2),
\end{aligned}
$$

together with

$$
\begin{aligned}
& g_{1}\left(r_{\ell}, x, y\right)=1-x+z+y(1-2 x)+r_{\ell} x(1-y)-r_{f} x z, \\
& h_{1}\left(r_{\ell}, x, y\right)=1-x+y+z(1-2 x)+r_{\ell} x(1-z)-r_{i} x y, \\
& g_{2}\left(r_{\ell}, x, y\right)=-2 x(1-y)+r_{\ell}(-1+x+x y)+r_{f} x z, \\
& h_{2}\left(r_{\ell}, x, y\right)=-2 x(1-z)+r_{\ell}(-1+x+x z)+r_{i} x y,
\end{aligned}
$$

where $\Lambda_{1}$ is given by (22), and $\Lambda_{2}=x+r_{\ell}(1-x)-r_{i} x y-r_{f} x z$. These expressions display a symmetry between $g_{p}$ and $h_{p}$, for a given $p$, under simultaneous interchange of $y, r_{i}$ and $z, r_{f}$. They reduce to

$$
\begin{aligned}
& g_{1}\left(r_{\ell}, x, y\right)=2+\left(r_{\ell}-2\right) x-\left(r_{\ell}+2\right) x y-r_{f} x z, \\
& h_{1}\left(r_{\ell}, x, y\right)=2-4 x+\left(r_{\ell}+2\right) x^{2}+\left(r_{\ell}+2-r_{i}\right) x y, \\
& g_{2}\left(r_{\ell}, x, y\right)=-r_{\ell}+\left(r_{\ell}-2\right) x+\left(r_{\ell}+2\right) x y+r_{f} x z, \\
& h_{2}\left(r_{\ell}, x, y\right)=-r_{\ell}+2 r_{\ell} x-\left(r_{\ell}+2\right) x^{2}-\left(r_{\ell}+2-r_{i}\right) x y .
\end{aligned}
$$

An obstacle to a simple analytic expression for the effective vertex is the $x$ integration in (24). However, if both initial and final quark masses are small compared with the gauge boson masss, i.e. if $r_{i}, r_{f} \ll 1$, one may consider making a linear approximation in $r_{i}$ and $r_{f}$ :

$$
\begin{aligned}
\frac{1}{\Lambda_{p}} & \approx \frac{1}{\Lambda_{p}^{(0)}}+\frac{1}{\left(\Lambda_{p}^{(0)}\right)^{2}} x\left(r_{i} y+r_{f} z\right) \quad(p=1,2) \\
\text { for } \Lambda_{1}^{(0)} & =1-x+r_{\ell} x, \quad \Lambda_{2}^{(0)}=x+r_{\ell}(1-x) .
\end{aligned}
$$

In this approximation of $\Lambda_{p}$, the functions $G_{p}$ and $H_{p}$ may be written as

$$
\begin{aligned}
& G_{p}=F_{p}\left(r_{\ell}\right)+r_{f} K_{p}\left(r_{\ell}\right)+r_{i} L_{p}\left(r_{\ell}\right), \\
& H_{p}=F_{p}\left(r_{\ell}\right)+r_{i} K_{p}\left(r_{\ell}\right)+r_{f} L_{p}\left(r_{\ell}\right) \quad(p=1,2),
\end{aligned}
$$


with a symmetry reflecting that between $g_{p}$ and $h_{p}$. Here $F_{p}\left(r_{\ell}\right), K_{p}\left(r_{\ell}\right)$, and $L_{p}\left(r_{\ell}\right)$ are functions of $r_{\ell}$ only. Writing $g_{p}=g_{p}^{(0)}+r_{f} g_{p}^{(1)}$ and $h_{p}=h_{p}^{(0)}+r_{i} h_{p}^{(1)}$, we see that $F_{p}$ comes from the $x y$ integral $\int g_{p}^{(0)} / \Lambda_{p}^{(0)}$ (or its equal $\int h_{p}^{(0)} / \Lambda_{p}^{(0)}$ ), while $K_{p}$ and $L_{p}$ arise from various terms linear in $r_{i}$ or $r_{f}$ in $g_{p}, h_{p}$, or $\Lambda_{p}$. From (28), together the functions $F_{p}\left(r_{\ell}\right), K_{p}\left(r_{\ell}\right)$, and $L_{p}\left(r_{\ell}\right)$ determine $G_{p}$ and $H_{p}$ for $p=1,2$ to first order in $r_{i}, r_{f}$. They are given by $(d=r-1)$

$$
\begin{aligned}
F_{1}(r)= & \frac{r}{4 d^{3}}\left(1-5 r-2 r^{2}\right)+\frac{3 r^{3}}{2 d^{4}} \ln r, \\
K_{1}(r)= & \frac{r}{72 d^{5}}\left(13-11 r-195 r^{2}+17 r^{3}-4 r^{4}\right) \\
& +\frac{r^{2}}{6 d^{6}}\left(-3+13 r+5 r^{2}\right) \ln r, \\
L_{1}(r)= & \frac{r}{36 d^{5}}\left(7-65 r-141 r^{2}+23 r^{3}-4 r^{4}\right) \\
& +\frac{r^{3}}{3 d^{6}}(13+2 r) \ln r, \\
F_{2}(r)= & \frac{r}{4 d^{3}}\left(-2-5 r+r^{2}\right)+\frac{3 r^{2}}{2 d^{4}} \ln r, \\
K_{2}(r)= & \frac{r}{72 d^{5}}\left(41-237 r+39 r^{2}-29 r^{3}+6 r^{4}\right) \\
& +\frac{r}{6 d^{6}}\left(-2+8 r+9 r^{2}\right) \ln r, \\
L_{2}(r)= & \frac{r}{18 d^{5}}\left(-5-105 r+33 r^{2}-16 r^{3}+3 r^{4}\right) \\
& +\frac{r}{6 d^{6}}\left(-1+22 r+9 r^{2}\right) \ln r .
\end{aligned}
$$

We have omitted in the above expressions terms that are numerical constants. Such terms independent of $r_{\ell}$ will give vanishing contributions as long as $F_{p}, K_{p}$, and $L_{p}$ are used under the sums carried out over all allowed $\ell$, as in Eq. (23), for $\sum_{\ell} \lambda_{\ell}=0$ by the unitarity of the CKM matrix.

It is crucial to note that for the down-type transitions such as $s \rightarrow d$ and $b \rightarrow s$, we have $Q_{1}=-1, Q_{2}=2 / 3$ and $\ell=1,2,3$ correspond respectively to $u, c, t$; whereas for up-type transitions $c \rightarrow u$ and $t \rightarrow c$, we have $Q_{1}=+1$, $Q_{2}=-1 / 3$, and $\ell=d, s, b$.

Inami and Lim [1] have entirely neglected the external masses in their calculations of $G_{p}$ and $H_{p}$ by taking $r_{i}=r_{f}=0$, so that $G_{p}=H_{p}=F_{p}$ in the general formula Eq. (23). For the stated purpose of their work, they have given the explicit expression for $-F_{1}+(2 / 3) F_{2}$ valid for the down-type transitions like $s \rightarrow d \gamma$ or $b \rightarrow s \gamma$; namely,

$$
-F_{1}+\frac{2}{3} F_{2}=\frac{r}{12(r-1)^{4}}\left[(1-r)\left(7-5 r-8 r^{2}\right)+6 r(2-3 r) \ln r\right]^{r \rightarrow 0} \rightarrow \frac{7}{12} r .
$$


But for the up-type transitions such as $c \rightarrow u \gamma$ (always within the $r_{i}=r_{f}=0$ approximation), one should use instead the expression

$$
F_{1}-\frac{1}{3} F_{2}=\frac{-r}{12(r-1)^{4}}\left[(1-r)\left(5-10 r-7 r^{2}\right)+6 r(1-3 r) \ln r\right] \stackrel{r \rightarrow 0}{\rightarrow} \frac{-5}{12} r
$$

and not the following combination [5], [6] or its limiting value [4]

$$
-F_{1}-\frac{1}{3} F_{2}=\frac{r}{12(r-1)^{4}}\left[(1-r)\left(1-10 r-5 r^{2}\right)-6 r(1+3 r) \ln r\right] \stackrel{r \rightarrow 0}{\longrightarrow} \frac{1}{12} r .
$$

As for the leptonic transitions listed in Table 1, the up-type heavy neutrino decay amplitude $\nu_{1} \rightarrow \nu_{2} \gamma$ having $Q_{1}=1, Q_{2}=-1$ is associated with the combination [8]

$$
F_{1}-F_{2}=\frac{3 r}{4(r-1)^{3}}\left(1-r^{2}+2 r \ln r\right),
$$

which differs from [9] by a constant 3/2. As noted before, when summation over $\ell$ is carried in the amplitude (23), this $r_{\ell}$-independent constant $3 / 2$ does not contribute however, due to $\sum_{\ell} \lambda_{\ell}=0$.

For the down-type $\tau^{-} \rightarrow \mu^{-} \gamma\left(Q_{1}=-1, Q_{2}=0\right)$ on the other hand, the corresponding amplitude is simply associated with $-F_{1}$.

To examine the relative importance of the external masses, we calculate the decay rates of $q_{i} \rightarrow q_{f} \gamma$, using the linear approximations in $r_{i}, r_{f}$, as defined in Eqs. (23)-(29). For this purpose, it is convenient to define the amplitudes

$$
\begin{aligned}
S_{p}(r) & =\frac{1}{2}\left[G_{p}(r)+\sqrt{r_{f} / r_{i}} H_{p}(r)\right], \\
P_{p}(r) & =\frac{1}{2}\left[G_{p}(r)-\sqrt{r_{f} / r_{i}} H_{p}(r)\right],
\end{aligned}
$$

so that Eq. (23), when summed over both groups of diagrams, may be rewritten as

$$
T_{\mu}=\frac{e G_{\mathrm{F}} M_{W} \sqrt{r_{i}}}{4 \sqrt{2} \pi^{2}}\left(A+B \gamma_{5}\right) i \sigma_{\mu \nu} q^{\nu},
$$

where

$$
\begin{aligned}
A & =\sum_{\ell=1}^{3} \lambda_{\ell} \sum_{p=1}^{2} Q_{p} S_{p}\left(r_{\ell}\right)=\sum_{\ell=1}^{2} \lambda_{\ell} \sum_{p=1}^{2} Q_{p}\left[S_{p}\left(r_{\ell}\right)-S_{p}\left(r_{1}\right)\right] \\
B & =\sum_{\ell=1}^{3} \lambda_{\ell} \sum_{p=1}^{2} Q_{p} P_{p}\left(r_{\ell}\right)=\sum_{\ell=1}^{2} \lambda_{\ell} \sum_{p=1}^{2} Q_{p}\left[P_{p}\left(r_{\ell}\right)-P_{p}\left(r_{1}\right)\right]
\end{aligned}
$$

In the last step we have made use of the unitarity of the CKM matrix to replace $\lambda_{1}$ with $-\left(\lambda_{2}+\lambda_{3}\right)$. In terms of these amplitudes the decay rate for $q_{i} \rightarrow q_{f} \gamma$ 
is given by

$$
\Gamma\left(q_{i} \rightarrow q_{f} \gamma\right)=\frac{G_{\mathrm{F}}^{2} \alpha}{64 \pi^{4}} M_{W}^{5}\left(\sqrt{r_{i}}\right)^{5}\left(1-\frac{r_{f}}{r_{i}}\right)^{3}\left(|A|^{2}+|B|^{2}\right) .
$$

The results of our calculations based on Eq. (38) are shown in Table 2 together with $\Gamma_{0}$, the corresponding values of the width when the factors $r_{i}$ and $r_{f}$ in the amplitudes are neglected. We have used $m_{u}=5 \mathrm{MeV}, m_{c}=1.5 \mathrm{GeV}$, $m_{t}=174 \mathrm{GeV}, m_{d}=11 \mathrm{MeV}, m_{s}=150 \mathrm{MeV}$, and $m_{b}=4.9 \mathrm{GeV}$ for the quark masses, $M_{W}=80 \mathrm{GeV}$ for the $W$ boson mass, and the central values of the CKM matrix elements as given in the Review of Particle Physics [10].

We see that, with one exception (the $t \rightarrow c \gamma$ case), generally $\bar{\Gamma} \approx \Gamma_{0}$, i.e. apart from the kinematic factor $\left(\sqrt{r_{i}}\right)^{5}$, the external masses can be safely neglected in calculating the width for $q_{i} \rightarrow q_{f} \gamma$. The reason is that the external mass correction functions $K_{p}$ and $L_{p}$ enter the amplitudes $S_{p}$ and $P_{p}$ respectively accompanied by the mass factors $\sqrt{r_{i} r_{f}}$ and $\left[\left(\sqrt{r_{i}}\right)^{3}+\left(\sqrt{r_{f}}\right)^{3}\right] /\left(\sqrt{r_{i}}+\sqrt{r_{f}}\right)$, which are much smaller than 1 , the coefficient of $F_{p}$ [cf. Eqs. (28) and (34)]. The exceptional case is the $t \rightarrow c \gamma$ transition. Since $r_{t} \approx 4.73$, neglecting the mass of the initial quark is not justified, and even keeping just terms linear in $r_{t}$ as in Eqs. (27), 28) is not enough and misleading: the approximation has broken down. However, the fact that $\Gamma \approx 100 \Gamma_{0}$ for this transition clearly indicates the importance of the effect of the external top mass. In Table 3 we list the contributions of the intermediate quarks $(d, s$, and $b)$ to the amplitudes $S=S_{1}-(1 / 3) S_{2}$ and $P=P_{1}-(1 / 3) P_{2}$ of the $t \rightarrow c \gamma$ transition for both $m_{c}=0$ and $m_{c}=1.5 \mathrm{GeV}$. These data show that the mass of the final quark is completely negligible in this case as well, and the difference between $\Gamma$ and $\Gamma_{0}$ for this transition can be entirely attributed to the top mass.

\begin{tabular}{lll}
\hline \hline Mode & $\Gamma_{0}(\mathrm{GeV})$ & $\Gamma(\mathrm{GeV})$ \\
\hline$s \rightarrow d \gamma$ & $7.48 \times 10^{-29}$ & $7.53 \times 10^{-29}$ \\
$b \rightarrow s \gamma$ & $5.06 \times 10^{-17}$ & $5.10 \times 10^{-17}$ \\
$c \rightarrow u \gamma$ & $1.50 \times 10^{-28}$ & $1.50 \times 10^{-28}$ \\
$t \rightarrow c \gamma$ & $4.51 \times 10^{-14}$ & $2.73 \times 10^{-12}$ \\
\hline \hline
\end{tabular}

Table 2: Widths for $q_{i} \rightarrow q_{f} \gamma$ transitions.

Before leaving this section we will make estimates of the branching fraction for the $c \rightarrow u \gamma$ transition (i.e. its inclusive rate scaled to that of the semileptonic decay $\left.c \rightarrow q l^{+} \nu\right)$. The correct expression $\bar{F}=F_{1}-(1 / 3) F_{2}$ which we use differs in magnitude and sign from the wrong combination $\bar{F}^{\prime}=-F_{1}-(1 / 3) F_{2}$ for the relevant values of the argument $r_{\ell}$. The QCD-uncorrected branching we have obtained, $B^{(0)}=3.90 \times 10^{-16}$, is about 28 times larger than an estimate [5] based on $\bar{F}^{\prime}$. To calculate the QCD corrections, one begins with the Wilson 
coefficients evaluated at the $W$ mass scale; in particular the coefficient

$$
c_{7}\left(M_{W}\right)=-\frac{1}{2}\left[\frac{\lambda_{s}}{\lambda_{b}} \bar{F}\left(r_{s}\right)+\bar{F}\left(r_{b}\right)\right]
$$

yields $2.065 \times 10^{-3}$, to be compared with $-0.414 \times 10^{-3}$ when it is calculated with $\bar{F}^{\prime}$. The Wilson coefficients are then evolved down from $M_{W}$ to the renormalization scale $\mu=m_{c}$ to give the effective coefficient $c_{7}^{\text {eff }}\left(m_{c}\right)$. The resulting QCD-corrected branching fraction obtained is reduced by $1 \%$ from Burdman et al.'s estimate [5]. The reason for the smallness of this effect is that $c_{7}\left(M_{W}\right)$ makes a very small contribution to $c_{7}^{\text {eff }}\left(m_{c}\right)$ which is completely dominated by $c_{2}\left(M_{W}\right)$. Nevertheless, the point being made is that the correct input functions $F_{p}$ or, even better, $G_{p}$ and $H_{p}$ are crucial for a proper evaluation of the Wilson coefficients.

\begin{tabular}{llll}
\hline \hline Quark & $S=P\left(m_{c}=0\right)$ & $S\left(m_{c} \neq 0\right)$ & $P\left(m_{c} \neq 0\right)$ \\
\hline$d$ & $6.12 \times 10^{-8}$ & $6.20 \times 10^{-8}$ & $6.05 \times 10^{-8}$ \\
$s$ & $8.12 \times 10^{-6}$ & $9.02 \times 10^{-6}$ & $8.81 \times 10^{-6}$ \\
$b$ & $5.95 \times 10^{-3}$ & $6.00 \times 10^{-3}$ & $5.90 \times 10^{-3}$ \\
\hline$\Gamma(\mathrm{GeV})$ & $2.7269 \times 10^{-12}$ & & $2.7273 \times 10^{-12}$ \\
\hline \hline
\end{tabular}

Table 3: Contributions to $t \rightarrow c+\gamma$ in the linear approximation in $m_{c}^{2}$ and $m_{t}^{2}$.

\section{MASS EFFECT IN THE TOP QUARK DECAY}

Since $m_{c} \ll m_{t}$, and $G_{p}$ and $H_{p}$ must be comparable in magnitude, the amplitude (23) reduces to $R M G_{p}$ and we need only to perform an accurate evaluation of the integrals

$$
G_{p}(r)=\int_{0}^{1} \mathrm{~d} x \int_{0}^{1-x} \mathrm{~d} y \frac{1}{\Lambda_{p}} g_{p}(r, x, y),
$$

where $\Lambda_{1}=1-x+r x-r_{i} x y$ and $\Lambda_{2}=x+r(1-x)-r_{i} x y$, with $r_{i}=r_{t}, r=r_{\ell}$, and $\ell=d, s, b$. After integration over $y$, which yields logarithm functions, the $x$ integration leads to

$$
\begin{aligned}
G_{1}(r)= & \frac{(2+r)}{2 r_{i}}+\frac{1}{r_{i}^{2}}\left[\left(r_{i}-1\right)(2-r)+r^{2}\right] J \\
& +\frac{1}{r_{i}^{2}}\left[2\left(1-r_{i}\right)+r\right]\left[\mathcal{L}_{2}(1-r)-\mathcal{L}_{2}\left(\frac{1}{\alpha}\right)-\mathcal{L}_{2}\left(\frac{1}{\beta}\right)\right], \\
G_{2}(r)= & \frac{-(2+r)}{2 r_{i}}+\frac{1}{r_{i}^{2}}\left[\left(r_{i}-1\right)(2-r)+r^{2}\right] J
\end{aligned}
$$




$$
+\frac{1}{r_{i}^{2}}\left[r\left(r_{i}-r-2\right)\right]\left[\mathcal{L}_{2}\left(1-\frac{1}{r}\right)-\mathcal{L}_{2}\left(\frac{1}{1-\alpha}\right)-\mathcal{L}_{2}\left(\frac{1}{1-\beta}\right)\right] .
$$

The parameters $\alpha, \beta$ are defined by

$$
\begin{aligned}
\alpha & =\frac{1}{2 r_{i}}\left(1+r_{i}-r-\sqrt{\Delta}\right), \quad \beta=\frac{1}{2 r_{i}}\left(1+r_{i}-r+\sqrt{\Delta}\right), \\
\Delta & =\left(1+r_{i}^{2}+r^{2}\right)-2\left(r_{i}+r+r_{i} r\right) .
\end{aligned}
$$

For the transition under discussion, they satisfy the conditions $0<\alpha<\beta<1$. $J$ stands for

$$
\begin{aligned}
J= & -1+\ln r_{i}+\frac{r}{1-r} \ln r+\alpha \ln \alpha+(1-\alpha) \ln (1-\alpha) \\
& +\beta \ln \beta+(1-\beta) \ln (1-\beta)+i \pi \frac{\sqrt{\Delta}}{r_{i}},
\end{aligned}
$$

and $\mathcal{L}_{2}$ is the dilogarithm (Spence integral) 11] represented by

$$
\mathcal{L}_{2}(x)=-\int_{0}^{x} \frac{\ln (1-t)}{t} \mathrm{~d} t
$$

which admits the expansion series $\mathcal{L}_{2}(x)=\sum_{k=1}^{\infty} x^{k} / k^{2}$ for $|x| \leq 1$.

With a top mass larger than the mass of the $W$ boson and the internal quark, $\Lambda_{1}$ and $\Lambda_{2}$ may change signs over the range of the $x, y$ integrations, and hence the arguments of the various logarithm functions that appear in the course of the integrations may become negative, and imaginary parts will arise. Physically they signal the presence of the intermediate states on the mass shell. Thus, for each internal quark $\ell=d, s, b$, the imaginary parts of $G_{p}\left(r_{\ell}\right)$ correspond to the real emission process $t \rightarrow W^{+}+q_{\ell}+\gamma$.

\begin{tabular}{cccc}
\hline \hline & $G_{1}$ & $G_{2}$ & $S$ \\
\hline$d$ & $0.84375-i 0.80176$ & $-0.19858+i 0.82598$ & $0.45497-i 0.53855$ \\
$s$ & $0.84375-i 0.80177$ & $-0.19860+i 0.82600$ & $0.45498-i 0.53855$ \\
$b$ & $0.84541-i 0.80377$ & $-0.20823+i 0.83341$ & $0.45742-i 0.54079$ \\
\hline \multicolumn{3}{c}{$\Gamma(\mathrm{GeV})=8.45 \times 10^{-13}$} \\
\hline \hline
\end{tabular}

Table 4: Values of $G_{1}$ and $G_{2}$ for $d, s$, and $b$ in $t \rightarrow c+\gamma$.

Values of $G_{1}\left(r_{\ell}\right), G_{2}\left(r_{\ell}\right)$, and $S\left(r_{\ell}\right)=\frac{1}{2}\left[G_{1}\left(r_{\ell}\right)-\frac{1}{3} G_{2}\left(r_{\ell}\right)\right]$ for relevant $r_{\ell}$ in $t \rightarrow c \gamma$ are recorded in Table 1 . They depend weakly on the mass of the internal quark, being dominated by the large mass of the initial top quark. Thus, the Glashow-Iliopoulos-Maiani mechanism works effectively to suppress 
the amplitude

$$
A=B=\frac{1}{2} \sum_{\ell=d, s, b} \lambda_{\ell}\left[G_{1}\left(r_{\ell}\right)-\frac{1}{3} G_{2}\left(r_{\ell}\right)\right] .
$$

In contrast, in the zeroth or first order approximation (in $\left.r_{i}, r_{f}\right)$ of the transition amplitudes, the functions $S\left(r_{\ell}\right)$ and $P\left(r_{\ell}\right)$ depend strongly on the internal quark mass, as shown in Table 3. The contribution of the intermediate $b$ quark is then overwhelmingly dominant and the GIM mechanism hardly operative. However, this conclusion turns out to be erroneous, as is clear from the data presented in Table 4 . Finally, the exact $G_{1}$ and $G_{2}$ scale as $r_{t}^{-1} \sim 1$ [cf. (41)-(42)], whereas their approximate values scale at best as $r_{t} r_{b} \sim r_{b} \sim 10^{-2}$ [cf. (28)-(29)], which explains the striking differences in magnitudes that we see in Tables 3 and 1.

With the amplitude given in (46), we find the width $\Gamma(t \rightarrow c \gamma) \approx 8.45 \times$ $10^{-13} \mathrm{GeV}$, which falls between the values presented in Table 2 based on the approximation $r_{i}=r_{f}=0$ and the linear approximation (38). In brief, the effect of the initial fermionic mass in $t \rightarrow c \gamma$ and, by extension, $\tau \rightarrow \mu \gamma$ is both subtle and substantial.

\section{CONCLUSIONS}

We have supplemented the Inami-Lim formula for the $s \rightarrow d \gamma$ effective vertex with an equally simple formula for the $c \rightarrow u \gamma$ vertex [Eq. (31)] under the same assumptions that the external fermionic masses are negligible. We then proceeded on to re-examine the external mass effects and obtain the corresponding formulas [Eqs. (23), (28)-(29)] valid to linear order in $M^{2}$ and $m^{2}$, the results of which are shown in Table 2. Since this approximation breaks down (cf. Table 3) in the cases such as $t \rightarrow c \gamma$, in which the initial fermion mass is much larger than any internal mass, we derive the exact one-loop formula for such transitions [Eqs. (41)- 445] ] and evaluate the corresponding rate of the $t \rightarrow c \gamma$ transition in Table 4 .

Given the importance of flavor-changing electromagnetic transitions in testing the standard model and probing new physics, it is essential to have reliable results for these processes at the lowest, one-loop level since they are the key components in building up more sophisticated calculations.

\section{ACKNOWLEDGMENTS}

The work of QHK was supported by the Natural Sciences and Engineering Research Council of Canada.

\section{References}

[1] T. Inami and C. S. Lim, Prog. Theor. Phys. 65, 297, (1981).

[2] N. Deshpande and G. Eilam, Phys. Rev. D 26, 2463 (1982). 
[3] S. Bertolini, F. Borzumati, and A. Masiero, Phys. Rev. Lett. 59, 180 (1987).

[4] Ch. Greub, T. Hurth, M. Misiak, and D. Wyler, Phys. Lett. B 382, 415 (1996).

[5] G. Burdman, E. Golowich, J-A. Hewett, and S. Pakvasa, Phys. Rev D 52, 6383 (1995).

[6] S. Fajfer and P. Singer, Phys. Rev. D 56, 4302 (1997); S. Fajfer, S. Prelovsek, and P. Singer, hep-ph/9808369.

[7] G. Buchalla, A. J. Buras, and M. Lautenbacher, Rev. Mod. Phys. 68, 1125 (1996).

[8] Q. Ho-Kim, B. Machet, and X. Y. Pham, hep-ph/9902442.

[9] P.B. Pal and L. Wolfenstein, Phys. Rev. D 25, 766 (1982).

[10] Review of Particle Physics, Eur. Phys. J. C 3, 1 (1998).

[11] M. Abramowitz and I. A. Stegun, Handbook of Mathematical Functions (Dover, New York 1965). 


\section{FIGURE CAPTIONS}

Fig. 1 Contributions to $q_{i} \rightarrow q_{f} \gamma$ vertex: emission from bosons.

Fig. 2 Contributions to $q_{i} \rightarrow q_{f} \gamma$ vertex: emission from internal quark.

Fig. 3 Emission from initial or final quark.

Fig. 4 Kinematic variables in one-loop diagrams. 


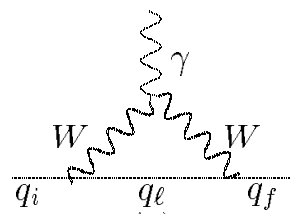

(a)

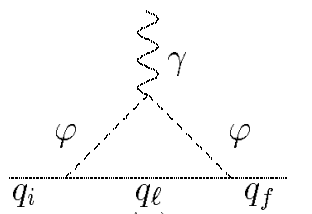

(b)

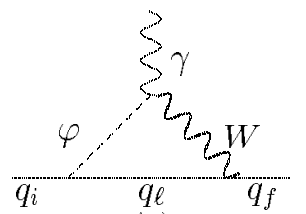

(c)

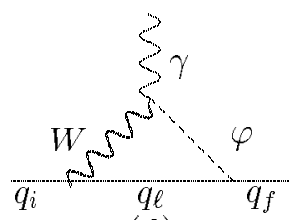

(d)

FIG. 1 Contributions to $q_{i} \rightarrow q_{f} \gamma$ vertex: emission from bosons

One-loop flavor changing electromagnetic transitions. Q. Ho-Kim \& X. Y. Pham 


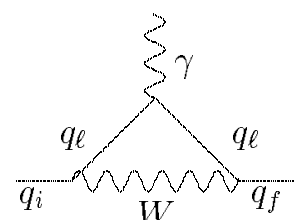

(a)

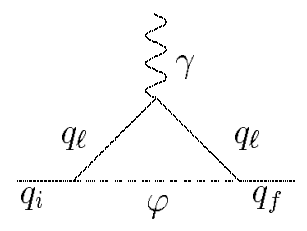

(b)

FIG. 2 Contributions to $q_{i} \rightarrow q_{f} \gamma$ vertex: emission from internal quark

One-loop flavor changing electromagnetic transitions. Q. Ho-Kim \& X. Y. Pham 

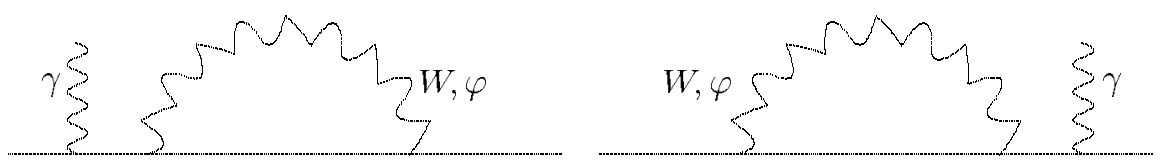

FIG. 3 Emission from initial or final quark

One-loop flavor changing electromagnetic transitions. Q. Ho-Kim \& X. Y. Pham 


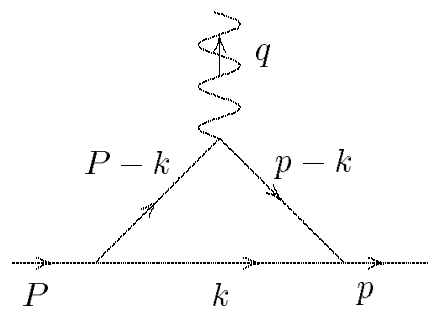

FIG. 4 Kinematic variables in one-loop diagrams

One-loop flavor changing electromagnetic transitions. Q. Ho-Kim \& X. Y. Pham 